\title{
Atividade física, propósito de vida de idosos ativos da comunidade: um estudo transversal
}

\author{
Physical activity, life purpose of community \\ active elderly people: a cross-section study
}

\author{
Tarcia Cristina Ferreira Duarte ${ }^{1}$ (1) \\ Higo da Silva Lopes ${ }^{2}$ (1) \\ Hércules Lázaro Morais Campos ${ }^{3}$ (1)
}

1,3Instituto de Saúde e Biotecnologia, Universidade Federal do Amazonas (Coari). Amazonas, Brasil. tarciacrisduarte@gmail.com, herculeslmc@hotmail.com

${ }^{2}$ Autor para correspondência. Instituto de Saúde e Biotecnologia, Universidade Federal do Amazonas (Coari). Amazonas, Brasil. higo.lopes17@gmail.com

\begin{abstract}
RESUMO | INTRODUÇÃO: A atividade física feita de maneira regular tem grande eficácia no retardo do declínio fisiológico do processo do envelhecimento, mantém e otimiza a capacidade física, melhora a funcionalidade e tem expressão no propósito de vida na velhice. OBJETIVO: Caracterizar e descrever as condições sociodemográficas, físicas e de propósito de vida em um grupo de idosos praticantes de atividade física regular no interior do Amazonas. MÉTODOS: Estudo transversal com idosos ativos da comunidade que realizam atividade física em suas comunidades por pelo menos 3 vezes na semana em torno de 50 minutos. Estes foram avaliados através de questionários e escalas validadas: Mini Exame Exame Estado Mental (MEEM), Teste de Trilha, Propósito de Vida, Teste Short PhysicalPerformanc e Battery (SPPB), Índice de Comorbidades Funcional, Escala numérica de dor e foram coletados dados referentes às características sociodemográficas. RESULTADOS: Dos 36participantes, $100 \%$ são naturais do interior do Amazonas; 28 (77\%) sobrevivem com renda mensal de salário mínimo; $27(75 \%)$ relatam sentir dor em alguma parte do corpo e $21(58,3 \%)$ apresentaram sobrepeso ou obesidade grau 1,33(91,6\%) diz se sentir bem quando pensam no passado e no futuro. CONCLUSÃO: Apesar de sentir dor, de baixa renda e baixa escolaridade,os idosos praticam atividade física que traz benefícios como manutenção da saúde física, diminuição dos sintomas depressivos, percepção de realização, motivação, satisfação e felicidade em viver.
\end{abstract}

PALAVRAS-CHAVE: Idoso. Atividade física em grupo. Motivação pessoal.

\begin{abstract}
INTRODUCTION: Physical activity performed on a regular basis is highly effective in delaying the physiological decline of the aging process, maintains and optimizes physical capacity, improves functionality and has an impact on the purpose of life in old age. OBJECTIVE: characterize and describe sociodemographic, physical and life purpose conditions in a group of elderly regular physical activity practitioners within the Amazon region. METHODS: Cross-sectional study with active elderly people who perform physical activity in their communities at least 3 times a week around 50 minutes. These were evaluated using validated questionnaires and scales: Mini Mental State Examination (MMSE), Trail Test, Life Purpose, Short Physical Performance and Battery Test (SPPB), Functional Comorbidity Index, Numerical Pain Scale and data were collected regarding sociodemographic characteristics. RESULTS: Of the 36 participants, $100 \%$ are from the interior of Amazonas state; 28 (77\%) survive on monthly minimum wage income; 27 (75\%) reported feeling pain in some part of the body and 21 (58.3\%) presented overweight or obesity grade; $1,33(91.6 \%)$ say they feel good when they think of the past and in the future. CONCLUSION: In spite of the grief of feeling pain, of low income and low education, the elderly engage in physical activity that is beneficial as physical maintenance, reduction in depressive symptoms and perceived motivation, satisfaction and happiness in living.
\end{abstract}

KEYWORDS: Aged. Physical activity in group. Personal motivation. 


\section{Introdução}

O envelhecimento é o processo biológico natural dinâmico e progressivo do ser humano causado por alterações na produção e armazenamento de componentes moleculares e bioquímicos acarretando mudanças morfológicas, fisiológicas, físicas e psicológicas que podem gerar várias disfunções, principalmente a perda da independência funcional do indivíduo e adaptação ao meio ambiente acarretando vulnerabilidades para adquirir agentes patológicos que podem levar à morte ${ }^{1-2}$.

O crescimento populacional de pessoas com 60 anos ou mais é um acontecimento mundial². No Brasil é possível observar a mudança no formato pirâmide etária, segundo a Pesquisa Nacional por Amostra de Domicílios Contínua - Características dos Moradores e Domicílios, houve um aumento do número de idosos de 4,8 milhões de 2012 para 2017, consistindo em $18 \%{ }^{3}$.No Amazonas, o índice populacional cresceu 3,5\% em dez anos, correspondendo a 8,8\% da população, com mais de 347 mil idosos ${ }^{4}$.

A atividade física feita de maneira regular tem grande eficácia no retardo do declínio fisiológico do processo do envelhecimento, mantendo e melhorando a qualidade de vida, função cardiovascular, força, capacidade funcional, massa muscular, capacidade, além de prevenir e controla doenças crônicas degenerativas e estados emocionais nocivos como depressão $0^{5-6}$.A atividade física regular é definida como um evento planejado, estruturado e repetitivo que tem como objetivo manter e melhorar aptidão física e os elementos que a integram ${ }^{5}$.

A velhice bem-sucedida envolve também fatores da saúde emocional do idoso verificando bem-estar psicológico que corresponde principalmente ao domínio de propósito de vida. Este refere-se aos sentimentos de que a sua existência tem sentido e direção, além de metas e planos são alcançáveis a curto, médio ou longo prazo. É uma percepção da vida do indivíduo sobre suaevolução pessoal, felicidades, satisfações, amor-próprio, autoconfiança, motivação para viver e realizar suas atividades de vidas ${ }^{?}$.
O objetivo deste trabalho foi caracterizar e descrever as condições sociodemográficas, físicas e de propósito de vida em um grupo de idosos praticantes de atividade física regular no interior do Amazonas.

\section{Materias e métodos}

Trata-se de um estudo transversal, realizado no município de Coari, no interior do Amazonas distante aproximadamente $350 \mathrm{~km}$ da capital do estado com uma população de 75.965 habitantes aproximadamente ${ }^{8}$. Este estudo foi aprovado pelo Comitê de Ética sob o parecer $n^{\circ}$ CAAE08021419.2.0000.5020.

A amostra foi composta por conveniência com idosos com idade entre 60 a 89 anos, participantes de um grupo de atividade física regular por pelo menos 3 vezes na semana por 50 minutos nas quadras ou pontos demarcados em seus bairros de acordo com Unidade de Saúde do Município de Coari- Amazonas. A coleta de dados foi realizada no período de agosto a setembro de 2019, no local que o idoso desejasse ser avaliado.

Os critérios de inclusão foram: idosos ativos e praticantes de atividade física regular; idade $\geq 60$ anos; deambulante com ou sem auxilio, estar residindo no município e aceitar participar da pesquisa.

Os critérios de exclusão foram: idosos sedentários, idade menor que 60 anos, incapacitados de deambular com ou sem auxilio, não estar residindo no municípioe não aceitar participar da pesquisa.

As informações foram coletadas por meio de questionário estruturado, teste cognitivo e teste físico aplicado por avaliadores treinados, respondido e realizado pelos próprios idosos. As variáveis analisadas foram:

1) Características sociodemográficas: nome, endereço, bairro, data de nascimento, idade, gênero, naturalidade e telefone.

2) Características socioeconômicas: escolaridade, anos de escolaridade, ocupação atual, renda mensal, mora só ou com quem. 
3) Características relacionada à saúde: a) condição de saúde: incontinência urinária, tontura, problemas de memória, problemas para dormir; b) número de medicamento de uso regular: medicamento e dose; c) uso de dispositivo assistido para marcha: sim ou não, se sim, bengala, andador, muleta; d) Sr. diria que sua visão/audição é: excelente, boa, regular, péssima; e) faz uso de aparelho auditivo: sim ou não;

4) Índice de Comorbidades Funcional: é uma escala composta por 18 comorbidades, ais quais o sujeito responde sim ou não caso possua?

5) Índice de Massa Corporal (IMC), calculado com base em informação auto referida de peso e altura ${ }^{10}$.

6) Avaliação cognitiva:

a) Mini Exame do Estado Mental: é um teste para avaliar cognição, com total de escore de 30 pontos, sendo que é composto de dois domínios agrupado em sete categorias: orientação para tempo, orientação para local, registro de três palavras, atenção e cálculo, recordação das três palavras, linguagem e praxiavisuo-construtiva. A escolaridade é um fator que influencia nos resultados para déficit cognitivo, por isso foram usadas as seguintes notas de corte de 20 para analfabetos, 25 com 1 a 4 anos de estudo, 27 de 5 a 8 anos, 28 de 9 a 11, 29-30 para 12 anos ou mais ${ }^{11-12}$.

b) Teste de Trilha Modificado 2B: é utilizado para avaliar função de atenção seletiva, capacidade de alternar o foco mesmo com estímulos de desatenção e flexibilidade mental. Na aplicação é solicitado ao sujeito ligar um número ao uma letra em ordem crescente (exemplo: 1-A, 2-B), se acerta todo o teste pontua, caso não, é referido com déficit cognitivo ${ }^{12}$.

7) Escala de Depressão Geriátrica: é uma escala composta de 15 questões com resposta sim ou não, o resultado é dado pela soma das pontuações, quanto mais alto o escore, maior a intensidade dos sintomas depressivos ${ }^{13-14}$.

8) Quantificação de dor numérica: a escala de dor numérica é utilizada para mensurar a dor em idosos, é considerada a mais fidedigna a ser aplicada a essa faixa etária, possui uma escala de 0 a 10, consistindo em
0 sem nenhuma dor e 10 a pior possível. Além disso, foi aplicada uma questão de onde seria local a dor $15-16$.

9) Capacidade Física: o Teste Short PhysicalPerformanc e Battery (SPPB) é indicado para avaliar a capacidade funcional do idoso e rastreio para déficits futuros pelos seguintes domínios: a velocidade da marcha em passo habitual, equilíbrio estático em posição ortostática, resistência e força dos membros inferiores por meio de observação se o idoso consegue levantar e sentar na cadeira ${ }^{17}$.

10) Propósito de vida: é um questionário com 10 questões para avaliar o estado psicológico do paciente em relação a satisfação global com a vida, objetivos realizados, autoestima, entre outros aspectos ${ }^{7}$.

Para o procedimento de análise foi produzida uma planilha eletrônica no programa Excel 2013 onde foram tabulados os dados e posteriormente foram exportados para software Package for Social Sciences (SPSS), versão 2010. Realizou-se análise descritiva por meio de distribuição das variáveis de interesse do estudo.

\section{Resultados}

A amostra foi composta de 36 idosos praticantes de atividade física, com idadeentre 60 e 89 anos, sendo prevalente a faixa etária 60 a 69 anos com 18(50\%), de baixa renda $31(86,1 \%)$, predominantemente mulheres $26(72 \%)$ e analfabetos $24(66,7 \%)$.As demais características da amostra estãoapresentadas na tabela 1.

As caracterizar o índice de massa corporal dos idosos, 21 (58,3\%) variam entre sobrepeso à obesidade grau 1 e 27 (75\%) relata sentir dor em alguma parte do corpo ainda assim não deixam de realizar os exercícios semanais. As demais características físicas estão descritas na tabela 2.

Avaliou-se o resultado do questionáriode propósito de vida em que $33(91,6 \%)$ dos idosos praticantes de atividade física regular afirmam sentir bem quando pensa no passado e no futuro e $25(69,4 \%)$ discorda que as atividades de vida diária não têm importância. Os demais dados estão descritos na tabela 3. 
Tabela 1. Caracterização Sociodemográficas dos idosos praticantes de atividade física regular em uma cidade do interior do Amazonas, 2019

\begin{tabular}{|c|c|c|}
\hline \multirow{2}{*}{$\begin{array}{l}\text { Variáveis } \\
\text { Idade } \mathbf{n}(\%)\end{array}$} & \multicolumn{2}{|c|}{$n=36$} \\
\hline & $\mathbf{n}$ & $(\% / \pm \mathrm{DP})$ \\
\hline Idosos jovens - 60 a 69 anos & 18 & $(50)$ \\
\hline Idosos velhos - 70 a 79 anos & 14 & $(38,9)$ \\
\hline Sexo feminino, $\mathrm{n}(\%)$ & 26 & (72) \\
\hline Escolaridade (analfabeto), média (DP). & 2,5 & $( \pm 1,79)$ \\
\hline Anos de escolaridade (0 a 5 anos), $n$ (\%) & 28 & $(77,7)$ \\
\hline Renda mensal (até um salário mínimo), n (\%). & 31 & $(86,1)$ \\
\hline Aposentado, n (\%) & 30 & $(83,3)$ \\
\hline Natural do interior do Amazonas, n (\%) & 36 & $(100)$ \\
\hline Não usa dispositivo de auxílio para marcha, n (\%) & 36 & $(100)$ \\
\hline Percepção subjetiva da visão regular ou péssima, n (\%) & 30 & $(83,3)$ \\
\hline Percepção subjetiva da audição boa, n (\%) & 16 & $(44,4)$ \\
\hline Mora com esposo (a) ou filho (a) n, (\%) & 28 & $(77,7)$ \\
\hline Faz uso de 2 a 4 medicações, n (\%) & 16 & $(44,4)$ \\
\hline Usa Anti-hipertensivo, n (\%) & 18 & $(50)$ \\
\hline \multicolumn{3}{|l|}{ MEEM (escore $\geq 20$ normal) } \\
\hline Não pontuou MEEM & 5 & $(13,9)$ \\
\hline Pontuou menos de 20 & 19 & $(52,8)$ \\
\hline Teste de trilha, média (DP) & 0,2 & $( \pm 0,4)$ \\
\hline Não acertou o teste de trilha $n(\%)$ & 28 & $(77,8)$ \\
\hline GDS ( $\geq 5$ Sintomas depressivos), $n$ (\%) & 20 & $(55,6)$ \\
\hline
\end{tabular}

MEEM (Mini Exame de Estado Mental), GDS (Escala de Depressão Geriátrica)

Tabela 2. Características físicas dos idosos praticantes de atividade física regula em uma cidade do interior do Amazonas, 2019

\begin{tabular}{ll}
\hline Variáveis & $\mathrm{n}=36$ \\
\hline Índice de comorbidades funcional $\mathbf{n}, \mathbf{( \% )}$ & $\mathbf{n}(\% \mathbf{m} \mathbf{D P})$ \\
1 a 2 Comorbidades. & $14(38,8)$ \\
IMC de sobrepeso para obesidade grau 1. & $21(58,3)$ \\
Impedimento visual. & $20(55,6)$ \\
SPPB, média (DP) & $7,5( \pm 2,2)$ \\
Dimensões do SPPB, média (DP) & \\
Equilíbrio & $3,7( \pm 0,6)$ \\
Velocidade de Caminhada & $1,8( \pm 1,0)$ \\
Força & $1,8( \pm 1,1)$ \\
Dor em alguma parte do corpo, $\mathbf{n}(\%)$ & $27( \pm 75,0)$ \\
Locais mais frequentes de dor, $\mathbf{n}(\%)$ & $7(19,4)$ \\
Joelho & $5(13,9)$ \\
Coluna lombar & $4(11,1)$ \\
Coluna dorsal & \\
Escala numérica de dor, $\mathbf{n}(\%)$ & $13(36,1)$ \\
4 a 6 de intensidade moderada. &
\end{tabular}

SPPB (Shont Physical Performace Battery); IMC (Índice de Massa Corporal). 
Tabela 3. Propósito de vida dos idosos praticantes de atividade física regular no interior do Amazonas, 2019

\begin{tabular}{lc}
\hline Variáveis & $\mathrm{n}=36$ \\
\hline Propósito de vida & $\mathbf{n}(\%)$ \\
Tenho senso de direção e propósito de vida (concordo) & $35(97,2)$ \\
Sinto-me bem quando penso no passado e futuro (concordo) & $33(91,6)$ \\
Sou uma pessoa ativa (concordo) & $33(91,6)$ \\
Faço planos para o futuro (concordo) & $30(83,3)$ \\
Tenho objetivos na vida (concordo) & $30(83,3)$ \\
Foco no presente (concordo) & $26(72,2)$ \\
Atividades de vida diárias banais (discorda) & $25(69,4)$ \\
Vivo um dia de cada vez (concordo) & $22(61,1)$ \\
Não faço metas, perda de tempo (concordo) & $19(52,7)$ \\
Sinto que eu já fiz tudo na vida (concordo) & $19(52,7)$
\end{tabular}

\section{Discussão}

A maioria dos idosos ativos desse estudo eram composto por mulheres, com baixa escolaridade com predomínio de analfabetos, que moram com algum familiar e são aposentadas corroborando com os dados do estudo demográfico realizado em sete cidades brasileiras com prevalência do sexo feminino, idosas que também vivem no interior, vivem com fiIho ou entre familiares e são aposentados ${ }^{18-19}$.

A frequência mais alta de mulheres em pesquisas, deve-se ao fato de que as mesmas são mais preocupadas com sua saúde ${ }^{2,11}$. Além de terem mais consciência que para ter uma qualidade vida necessitam praticar atividade física ${ }^{19}$.

O baixo nível de escolaridade e poder aquisitivo dos idosos ativos do interior do Amazonas desse estudo não foi empecilho para a pratica de atividade física regular, visto que mais da metade dos idosos praticantes de atividades regulares são analfabetos ou com poucos anos de escolaridade e vivem com renda mensal de um salário mínimo, tal resultado foi demostrado diferentemente em uma revisão de literatura no Brasil realizada por Maciel ${ }^{5}$, onde baixo nível socioeconômico e escolaridade foram os principais motivos para que idosos não praticassem exercícios diferente dos idosos desse estudo que apesar possuírem essas características praticam atividade física.

Mesmo praticando atividade física regular os idosos desse estudo apresentaram pontuação ruim quando foram avaliados cognitivamente, contrariando o que é encontrado na literatura que idosos praticantes de atividade física associado à boa escolaridade na zona urbana pontuam melhor quando são avaliados pelo MEEM ${ }^{21}$. Ressalta-se novamente que os idosos desse estudo possuem baixa escolaridade com predomínio de analfabetos.

O presente estudo demonstrou que os idosos ativos praticantes de atividade física possuem sintomas depressivos. Sabe-se que a atividade física regular interfere de maneira positiva melhorando os sinais depressivos e de depressão e promove bem estar $4,5,20$.

A hipertensão é relatada na literatura como a comorbidade mais prevalente em idosos praticantes de atividade física regular, o que muitas vezes levam esses idosos a serem eletivos para o exercício através da UBS $19-21$. 
A dor é uma queixa comum em idosos que pode reduzir a qualidade de vida, pois promove fragilidade, insegurança ameaçando sua autonomia e independência, limitando a prática de suas atividades de vida diária e sociais ${ }^{15,22}$.

É recorrente na literatura a menção de dor crônica por mais de 6 meses, prevalentesnos locais de coluna lombar, coluna dorsal, região da pernae articulação do joelho ${ }^{15,23}$.Segundo Dellaroza MS et al. ${ }^{23}$ entre os fatores mais citados como desencadeantes de dor em idosos estão as atividades físicas e quanto maior os sintomas depressivos maior a referência de intensidade da dor.

Em um estudo realizado por Ferreti $\mathrm{F}^{24} \mathrm{com} 385$ idosos apontou uma correlação fraca de que o nível de atividade física diminua quando o idoso tem dor e doenças crônicas associadas, observou-se ainda que idosas que possuem dor crônica realizam menos atividade física.

Ao analisarmos os resultados de pontuações do SPPB isoladamente os idosos desse estudo apresentam melhor desempenho no equilíbrio estático quanto comparado ao equilíbrio dinâmico. Alterações no equilíbrio, velocidade da marcha e diminuição de força em membros inferiores são predisposições para maior risco de queda, para estes idosos que possuem essas características é recomendado a pratica de atividade física como prevenção ${ }^{17}$.

Os resultados do questionário de propósito de vida aplicado aos idosos do interior do Amazonas foram acima da média, com pontuações altas para bem-estar psicológico, autoestima, direção e sentido de vida, metas e objetivos que pretendem realizar, crescimento pessoal motivação para viver, possuindo uma visão positiva da vida e percepção de felicidade. Além disso, a baixa renda e escolaridade não influenciaram na redução do resultado. Segundo Ribeiro CC et al.? em um estudo sobre propósito de vida com 187 idosos observou-se que quanto maior a renda melhor era propósito de vida e quanto maior a escolaridade melhor o bem-estar psicológico desse idoso, pois se propõe a usar suas capacidades funcionais para atingir suas metas e objetivos.
A idade influência no sentido e direcionamento dos objetivos da vida, pois os idosos mais jovem com idade menor que 80 anos estão mais dispostos fisicamente e mentalmente, sendo assim conseguem cumprir suas metas desejadas, já os que estão na fase mais avançada, na maioria das vezes encontram-se mais frágeis, por isso tendem a não criar tantos planos e ter objetivos, pois avaliam ser inalcançáveis. O presente estudo possui um número maior de idosos com idade ente 60 a 79 anos permitindo um resultado mais alto na avaliação de propósito de vida ${ }^{7}$.

A melhor maneira encontrada para lidar com a incapacidade da velhice é ter um bom suporte social, pois isso ameniza o impacto das moléstias e tensões imprevistas, possibilitam aumentar a autoestima e o senso de controle com a vida e ambiente ${ }^{25}$.

Os idosos praticantes de atividade física regular na cidade de Coari ao serem abordados relatavam ter falta de tempo para serem avaliados para esse estudo, isso impactou diretamente no tamanho da amostra. A baixa escolaridade foi outro fator que dificultava para o idoso no momento de responder aos questionamentos.

\section{Conclusão}

Os idosos ativos do interior do Amazonas apresentam baixa escolaridade, baixa renda, déficit cognitivo moderado, são a grande maioria hipertensos, relatam sentir dor de moderada a intensa em alguma parte do corpo, apresentam baixo desempenho físico, principalmente em suas funções dinâmicas. Contudo, tais fatores não são barreiras para que os idosos pratiquem atividade física regular, além de não influenciarem em seu propósito de vida, já que os mesmosse sentem realizados, motivados, satisfeitos e felizes, visto que estes possuem maior suporte social por terem seus familiares e amigos mais próximos e presentes pelo fácil acesso, o que pode ser o provedor do bem-estar social e crescimento pessoal. 


\section{Contribuições dos autores}

Duarte TCF participou da concepção, revisão de literatura, coleta de dados, análise estatística, interpretação dos resultados, redação e revisão do texto. Lopes HS participou da revisão de literatura, interpretação dos resultados, redação e revisão crítica do texto. Campos HLM participou da concepção, análise estatística, interpretação dos resultados e orientou o trabalho.

\section{Conflitos de interesses}

Nenhum conflito financeiro, legal ou político envolvendo terceiros (governo, empresas e fundações privadas, etc.) foi declarado para nenhum aspecto do trabalho submetido (incluindo, mas não se limitando a subvenções e financiamentos, participação em conselho consultivo, desenho de estudo, preparação de manuscrito, análise estatística, etc.).

\section{Referências}

1. Mazo GZ, Liposcki DB, Ananda C, Prevê D. Condições de saúde, incidência de quedas e nível de atividade física dos idosos. Rev Bras Fisiot. 2007;11(6):437-42. doi: 10.1590/S141335552007000600004

2. Oliveira DV, Lima MCC, Contessoto LC, Cremonez JC, Antunes MD, Nascimento Junior JRA. Fatores associados ao nível de atividade física de idosos usuários das academias da terceira idade. Acta Fisiatr. 2017;24(1):17-21.

3. Instituto Brasileiro de Geografia e Estatística. Pesquisa Nacional Por Amostra de Domicílios Contínua. Número de idosos cresce 18\% em 5 anos e ultrapassa 30 milhões em 2017 [Internet]. 2018. [acesso em 2019 out 10]. Disponível em: https://bit.ly/2RdaM6U

4. G1 Amazonas. População Idosa no AM cresce 3,5\% em 10 anos, aponta IBGE Internet]. 2016 [acesso em 2019 out 10]. Disponível em: https://glo.bo/35kSskT

5. Maciel MG. Atividade física e funcionalidade do idoso. Motriz: Rev Educ Fis. 2010;16(4): 1024-32. doi: 10.5016/1980-6574.2010v1 6n4p1024

6. Mota J, Ribeiro JL, Carvalho J, Matos MG. Atividade física e qualidade de vida associada à saúde em idosos participantes e não participantes em programas regulares de atividade física. Rev Bras Educ Fís. 2006;20(3):219-25. doi: 10.1590/S180755092006000300007

7. Ribeiro CC, Neri AL, Yassuda MS. Semantic-cultural validation and internal consistency analysis of the Purpose in Life Scale forbrazilian older adults. Dement Neuropsychol. 2018; 12(3):24449. doi: $10.1590 / 1980-57642018 \mathrm{dn} 12-030004$
8. Instituto Brasileiro de Geografia e Estatística. Pesquisa Nacional Por Amostra de Domicílios Contínua. IBGE/Brasil/Amazonas/ Cidades/Coari [Internet]. 2010 [acesso 2019 out 10]. Disponível em: https://cidades.ibge.gov.br/brasil/am/coari/panorama

9. Marques WV, Cruz VA, Rego J, Silva NA. Influência das comorbidades na capacidade funcional de pacientes com artrite reumatoide. Rev Bras Reumatol. 2016;56(1):14-21. doi: 10.1016/j. rbr.2015.01.009

10. Borim FSA, Barros MBA, Neri AL. Autoavaliação da saúde em idosos: pesquisa de base populacional no Município de Campinas, São Paulo, Brasil. Cad Saúde Pública. 2012; 28(4):769-80. doi: 10.1590/S0102-311X2012000400016

11. Brucki SMD, Nitrini R, Caramelli P, Bertolucci PH, Okamoto IH. Sugestões para uso de Mini-Exame de Estado Mental no Brasil. Arq Neuropsiquiatr. 2003;61(3-B):777-781. doi: 10.1590/S0004$\underline{282 \times 2003000500014}$

12. Mota MMPE, Banhato EFC, Silva KCA, Cupertino APFB. Triagem cognitiva: comparações entre o mini-mental e o teste de trilhas. Rev Estud de Psicol. 2008; 25(3):353-59. doi: 10.1590/S0103166X2008000300004

13. Andrade LP. Funções cognitivas frontais e controle postural na doença de Alzheimer: efeitos do programa de intervenção motora com tarefa dupla [tese]. Rio Claro: Universidade Estadual Paulista; 2011.

14. Alvarenga MRM, Oliveira MAC, Fanccenda O. Sintomas depressivos em idosos: análise dos itens da Escala de Depressão Geriátrica. Rev Acta Paul Enferm. 2012;25(4):497-503. doi: $\underline{10.1590 / \text { S0103-21002012000400003 }}$

15. Celich KLS, Galon C. Dor crônica em idosos e sua influência nas atividades da vida diária e convivência social. Rev Bras Geriatr Gerontol. 2009;12(3):345-59.

16. Andrade FA, Pereira LV, Sousa FAEF. Mensuração da dor no idoso: uma revisão. Rev Latino- Am Enf. 2006;14(2):271-76. doi: 10.1590/S0104-11692006000200018

17. Silva TO, Freitas RS, Monteiro MR, Borges SM. Avaliação da capacidade física e quedas em idosos ativos e sedentários da comunidade. Rev Soc Bras Clin Med. 2010;8(5):392-8.

18. Neri LA, Yassuda MS, Araújo LF, Eulálio MC, Cabral BE, Siqueira MEC et al. Metodologia e perfil sociodemográfico, cognitivo e de fragilidade de idosos comunitários de sete cidades brasileiras: Estudo FIBRA. Cad Saúde Pública. 2013;29(4):778-92. doi: 10.1590/ $\underline{\text { S0102-311X2013000400015 }}$

19. Batista NNLAL, Vieira DJN, Silva GMP. Caracterização de idosos participantes de atividade física em um centro de convivência de Teresina-PI. Enfermagem em Foco. 2012;3(1):07-11. doi: 10.21675/2357-707X.2012.v3.n1.212 
20. Cheik NC, Reis IT, Heredia RAG, Ventura ML, Tufick S, Antunes HKM et al. Efeitos do exercício físico e da atividade física na depressão e ansiedade em indivíduos idosos. R Bras Ci e Mov. 2003;11(3):45-52. doi: 10.18511/rbcm.v11i3.509

21. Dias RG, Streit IA, Sandreschi PF, Benedetti TRB, Mazo GZ. Diferenças nos aspectos cognitivos entre idosos praticantes e não praticantes de exercício físico. J Bras Psiquiatr. 2013;63(4):326-31. doi: 10.1590/0047-2085000000041

22. Campos HLM, Liebano RE, Lima CA, Perracini MR. Multidimensional investigation of chronic pain experience and physical functioning following hip fracture surgery: clinical implications. British Journal of Pain. 2019;1-8. doi: 10.1177/2049463719861994
23. Dellaroza MSG, Furuya RK, Cabrera MAS, Matsuo T, Trelha C, Yamanda KN et al. Caracterização da crônica e métodos analgésicos utilizados por idosos da comunidade. Rev Assoc Med Bras. 2008;54(1):36-41. doi: 10.1590/S0104-42302008000100018

24. Ferretti F, Silva MR, Pegoraro F, Baldo JE, Sá CA. Dor crônica em idosos, fatores associados e relação com o nível e volume de atividade física. BrJP. 2019;2(1):3-7. doi: 10.5935/25950118.20190002

25. Rabelo DF, Neri AL. Recursos psicológicos e ajustamento pessoal frente à incapacidade funcional na velhice. Psicol Estud. 2005;10(3):403-12. doi: 10.1590/S1413-73722005000300008 